\title{
A New Method for Controlling Mobility Management Cost of PatHO-LEO Satellite and Mobile IP Network
}

\author{
Debabrata Sarddar \\ $\mathrm{PhD}$ Scholar, \\ Department of E.T.C.E \\ Jadavpur University \\ Kalyan Kumar Das \\ M.Tech (2011) \\ Department of C.S.E \\ Jadavpur University
}

\author{
Soumya Das \\ Department of E.T.C.E \\ Bengal Institute of tec.WBUT \\ Sougata Chakraborty \\ M.Tech (2011) \\ Department of C.S.E \\ Jadavpur University
Mrinal Kanti Naskar
Jadavpur University \\ Drpartment ETCE
}

\author{
Dipsikha Ganguli \\ Department of E.T.C.E \\ B.P.Poddar I.M.T.,WBUT \\ Kunal Hui \\ M.Tech (2011) \\ Department of C.S.E \\ Haldita Institute of Technology, \\ WBUT
}

\begin{abstract}
In low earth orbit(LEO) satellite network Mobility management is one of the key technologies. The aim of mobility management is to track where the subscribers are, allowing calls, SMS and other mobile phone services to be delivered to them. In this paper, we have proposed an idea of controlling the frequency hops and hence controlled the mobility management cost of patHO-LEO and Mobile IP network.
\end{abstract}

\section{General Terms}

Satellite and Mobile IP Network

\section{Keywords}

Mobility management, handover, LEO satellite networks, wideband receiver

\section{INTRODUCTION}

The trend in designing future global communication systems is to provide fast and low cost service to all users any time. Modern terrestrial wireless networks such as mobile networks provide communication to a limited geographical area. In order to provide global coverage satellite networks can be very useful with terrestrial networks. So the application of satellite networks extends from traditional telephone and TV broadcast service to data service such as file downloading and uploading and internet browsing.

There are mainly four types of satellite communication system exists depending upon the types of satellites

Geostationary Satellite (GEO)

Medium orbit satellite (MEO)

Low earth orbit satellite (LEO) although mixed constellations exists.

\section{LOW-EARTH ORBIT (LEO)}

LEO systems fly about 1,000 kilometers above the Earth (between 400 miles and 1,600 miles) and, unlike GEOs, travel across the sky. A typical LEO satellite takes less than two hours to orbit the Earth, which means that a single satellite is "in view" of ground equipment for a only a few minutes. As a consequence, if a transmission takes more than the few minutes that any one satellite is in view, a LEO system must "hand off" between satellites in order to complete the transmission. In general, this can be accomplished by constantly relaying signals between the satellite and various ground stations, or by communicating between the satellites themselves using "inter-satellite links." [1]

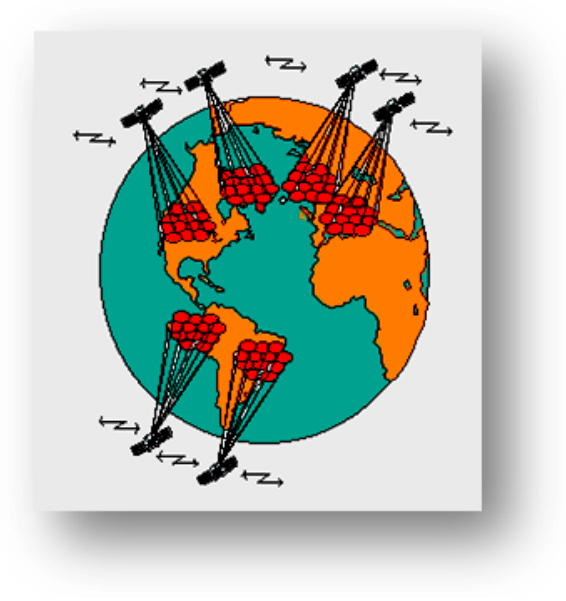

Figure 1 - LEO Satellite Cell Structure

In addition, LEO systems are designed to have more than one satellite in view from any spot on Earth at any given time, minimizing the possibility that the network will lose the transmission. Because of the fast-flying satellites, LEO systems must incorporate sophisticated tracking and switching equipment to maintain consistent service coverage. The need for complex tracking schemes is minimized, but not obviated, in LEO systems designed to handle only short-burst transmissions.

\section{Benefits of the LEO concept}

- LEOs can offer a communications infrastructure to areas where there is insufficient population to justify a terrestrial based cellular network. This not only includes many developing countries but $80 \%$ 
of the US as well. This explains why most of the initiatives for LEOs has come from the USA

- Many developing countries are interested in LEO systems as an alternative to investing in an very expensive terrestrial tele-communications infrastructure.

- Communication via LEOs does not suffer from the objectionably long transmission delays associated with geosynchronous systems.

- User equipment does not require high-power transmitters or highly directional antennae that need to be continually pointed to the satellite. In practice, transmit powers can be much lower than 1 watt.

- LEO satellites, are technically much simpler and more robust than geo-synchronous satellites and are less likely to suffer catastrophic failure during deployment or during the satellite lifetime. [2]

There are two types of LEO systems, Big LEOs and Little LEOs, each describing the relative mass of the satellites used as well as their service characteristics.

Little LEO satellites are very small, often weighing no more than a human being, and use very little bandwidth for communications. Their size and bandwidth usage limits the amount of traffic the system can carry at any given time. However, such systems often employ mechanisms to maximize capacity, such as frequency reuse schemes and load delay tactics.

Little LEO systems support services that require short messaging and occasional low-bandwidth data transport, such as paging, fleet tracking and remote monitoring of stationary monitors for everything from tracking geoplatonic movements to checking on vending machine status. The low bandwidth usage may allow a LEO system to provide more cost effective service for occasional-use applications than systems that maximize their value based on bulk usage. Examples of Little LEO systems include Orbcomm, Final Analysis and Leo One.

Big LEO systems are designed to carry voice traffic as well as data. They are the technology behind "satellite phones" or "global mobile personal communications system" (GMPCS) services now being developed and launched.

Most Big LEO systems also will offer mobile data services and some system operators intend to offer semi-fixed voice and data services to areas that have little or no terrestrial telephony infrastructure. Smaller Big LEO constellations also are planned to serve limited regions of the globe. Examples of Big LEO systems include Iridium, Globalstar and the regional Constellation and ECO-8 systems.

An emerging third category of LEO systems is the so-called "super LEOs" or "mega LEOs," which will handle broadband data. The proposed Teledesic and Skybridge systems are examples of essentially Big LEO systems optimized for packet-switched data rather than voice. These systems share the same advantages and drawbacks of other LEOs and intend to operate with inter-satellite links to minimize transmission times and avoid dropped signals. [3]

\section{Summary of LEO Pros and Cons}

- PRO: The transmission delay associated with LEO systems is the lowest of all of the systems.
- CON: The small coverage area of a LEO satellite means that a LEO system must coordinate the flight paths and communications hand-offs a large number of satellites at once, making the LEOs dependent on highly complex and sophisticated control and switching systems.

- PRO: Because of the relatively small size of the satellites deployed and the smaller size of the ground equipment required, the Little LEO systems are expected to cost less to implement than the other satellite systems discussed here.

- CON: LEO satellites have a shorter life span than other systems mentioned here. There are two reasons for this: first, the lower LEO orbit is more subject to the gravitational pull of the Earth and second, the frequent transmission rates necessary in LEO systems mean that LEO satellites generally have a shorter battery life than others. [4]

Comparing with other systems, LEO satellite systems is most preferable because of its different advantages such as low propagation delay, low handoff latency, low power requirement and effective bandwidth utilization. But is some disadvantage also. The main disadvantage is the speed of the satellite is very high than MN's and earth's speed. So the handover occurrence is more and the system design becomes more complex.

Handovers may degrade the system performance as a unsuccessful handover results call blocking and forced call termination. Forced call termination is less desirable than a new call blocking though both affect the performance of the system. A number of handover techniques have been proposed to solve this problem[5].

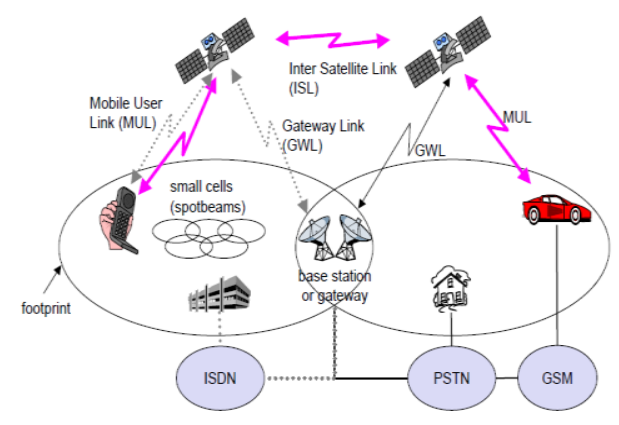

Figure 2: Satellite Communication Architecture

Handoff latency is also affected of the system .there are some method of reducing the handoff latency of the system [7][8][9].Handoff failure is one of the big issue during handover management.Some methods are discussed toovercome these in [11].These not has been discussed here.

In this paper we have proposed a new technique for controlling the mobility management cost during handover. We have proposed the idea of controlling the frequency hops.

The paper is organised as follow: in the second section we have described the related works on handover management. In the third section we have described the details of our work proposed work. In the forth section the simulation results of both our method and standard methods. In the next section we conclude the whole paper and finally a future work is mention regarding this paper in section six. 


\section{RELATED WORK}

The most widely used protocol for handover in satellite is MIP [12]. It is proposed by The Internet engineering task force (IETF) to handle mobility of internet hosts for mobile data communications. MIP is based over the concept of Home Agent (HA) and Foreign Agent (FA) for delivering of packets from one $\mathrm{MN}$ to $\mathrm{CN}$. It is basically completed by following steps:

When handover begins MN registers itself in FA and waits for allocation of channels in FA and updates its location in HA directory. HA encapsulates the data packets sent to it. Then encapsulated packets are sent to The FA.FA de-capsulate those packets and sent it to $\mathrm{MN}$.

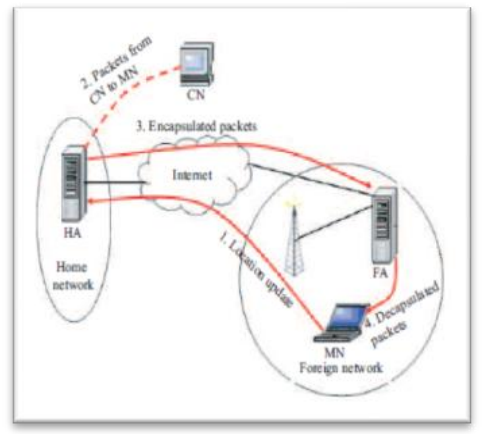

Figure 3: Handover Flow of Mobile IP

Another method is Seamless handover management scheme [13] (SEAHO-LEO) proposed by Aysegul et al in 2006[14]

It reduces packet loss and handover latency. It is describes in following steps.

First is to calculate a new IP. Then Send handover preparation request to current satellite Start to use new IP to send data packets \& CN starts to use new satellite

SEAHO-LEO provides efficient utilization of network bandwidth because of the absence of tunnelling and also does not need any change in existing internet infrastructure.

The main disadvantage of this process is high messaging traffic.

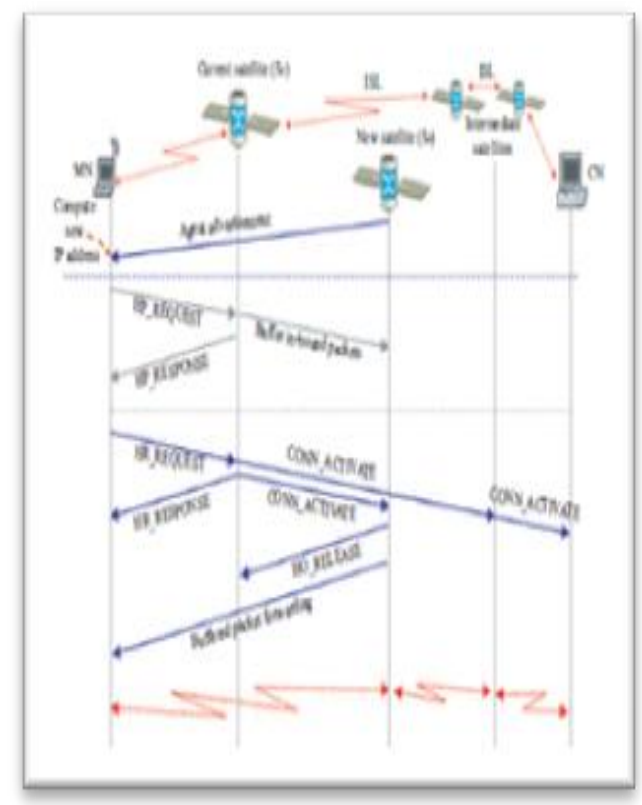

Figure 4: Signalling Flow of SeaHO-LEO
Another method to remove high messaging traffic is Pattern based handover management. It describes as follows

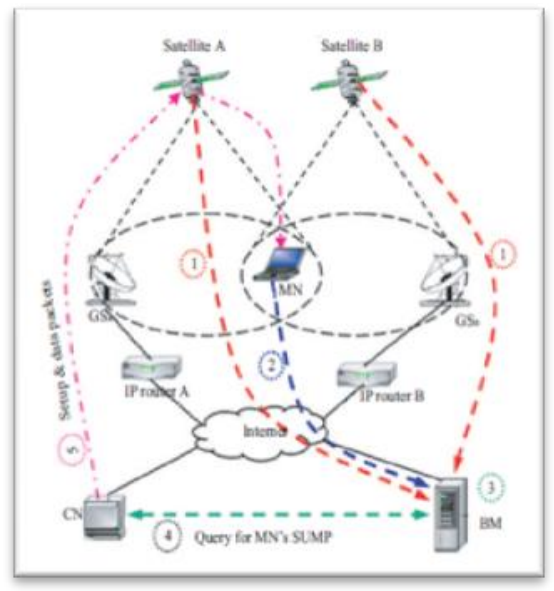

Figure 5: Handover scenario in PatHO-LEO

Satellites register to $\mathrm{BM}$. Then $\mathrm{MN}$ registers to $\mathrm{BM} \&$ establishes the satellite and user mobility pattern (SMUP) table. Then $\mathrm{CN}$ and $\mathrm{BM}$ establish connection and $\mathrm{CN}$ sends data packets to $\mathrm{MN}$.

There are other mobility management protocols like Transport layer seamless handoff schemes for space networks (TraSHSN) [15], paging in mobile IP (P-MIP) [16], and cellular IP [17]. These methods are not covered in this paper.

\section{B. Analysis of Mobility Management Cost of MIP \& PatHO-LEO}

In paper [13], the handover management cost of PatHO-LEO protocol has been analyzed and compares it to that of mobile IP. In [14] more detailed simulation results of PatHO-LEO can be found the handover management cost consists mainly of the cost of binding update and data delivery. The management cost is computed as the product of the generated control message size, $\mathrm{M}$, and the number of hops, $\mathrm{H}$, required to deliver the message. Equation (1) indicates the definition of the cost in this evaluation.

Cost $=$ M.H.... (1)

Now,

$$
\begin{gathered}
\mathbf{C}_{\mathrm{MIP}}(\mathbf{t})=\mathbf{M} \cdot{ }_{\mathrm{HMN}, \mathrm{LD}} \mathbf{R}_{\mathrm{HO}}(\mathbf{t}) \ldots(\mathbf{2}) \\
\mathbf{R}_{\mathrm{HO}}(\mathbf{t})=\text { VsatLsat } \int_{\text {Vsat }(\mathrm{t}-\ldots \mathrm{t})}^{\text {Vsatt }} D l(\text { Vsat }) t \mathrm{dt} \ldots . .(3)
\end{gathered}
$$

where, $\mathrm{V}_{\text {sat }}$ and $\mathrm{L}_{\mathrm{sat}}$ denote the ground speed of satellite and the coverage boundary length, respectively. $\mathrm{D}_{\mathrm{L}}\left(\mathrm{V}_{\text {sat }} \mathrm{t}\right)$ is the linear density of nodes on the coverage of satellite at time t. In our evaluation, we calculated nodes density as the ratio of the total number of nodes to the coverage surface area. In the PatHO-LEO model, the local forwarding and paging scheme create some additional cost. The total cost of PatHO-LEO model, $\mathbf{C}_{\text {Patho-Leo(t), is }}$

$$
\begin{aligned}
& \mathrm{C}_{\mathrm{PatHO}-\mathrm{LEO}}(\mathrm{t})=\mathrm{M} \cdot \mathrm{H}_{\mathrm{MN}, \mathrm{LD}}+\mathrm{M} \cdot \mathrm{H}_{\mathrm{AR}, \mathrm{AR}} \mathrm{R}_{\mathrm{HO}}(\mathrm{t}) \alpha+ \\
& \left\{\mathrm{M} \cdot \mathrm{H}_{\mathrm{AR}, \mathrm{AR}}(\mathrm{S}-\mathbf{1})+\mathrm{M} \cdot \mathrm{S}\right\} \times \mathbf{n}(\mathrm{t})(\mathbf{1}-\alpha) \lambda \ldots \text { (4) }
\end{aligned}
$$

where,

$\mathrm{n}(\mathrm{t})=$ the total number of MNs per coverage area at time $\mathrm{t}$ $\alpha=$ the ratio active MNs to the total number of MNs. 
$\mathrm{H}_{\mathrm{AR}, \mathrm{AR}}=$ the number of hops between two adjacent satellites $\mathrm{S}=$ the number of single-beam satellites that cover a single paging area.

\section{$\lambda=$ The rate of new connections to a $\mathrm{MN}$.}

The first term in (4) indicates the handshaking cost of PatHOLEO. Since all binding update operations are done at the beginning of the communication, there is no binding cost during handover in the PatHO-LEO. The second and third terms represent the local forwarding and paging costs, respectively. In the third term, the

expression $n(t)(1-\alpha) \lambda$ denotes the occurrence rate of paging. Since a satellite is required to send a paging request to its $(\mathrm{S}-$ 1) neighboring satellites, the cost of sending these requests is $\mathrm{M} \cdot \mathrm{H}_{\mathrm{AR}, \mathrm{AR}}(\mathrm{S}-1)$. Then, each satellite broadcasts the paging messages to the MNs within its coverage area. The cost is M.1.S for this operation.

Now for controlling the cost we have proposed an idea of controlling the number of hops between the MN and a location directory using a dual hopping wideband receiver. Here also controlling the term $\mathrm{H}_{\mathrm{MN}, \mathrm{LD}}$ we can control the cost of MIP and also PatHO-LEO.

\section{PROPOSED WORK}

In this paper we have proposed an idea of implementing a dual hopping wideband receiver for the mobility management cost of PatHO-LEO. From previous papers we have got the equations of cost

$$
\begin{aligned}
& \mathbf{C}_{\mathrm{MIP}}(\mathbf{t})=\mathbf{M} \cdot \mathbf{H}_{\mathrm{MN}, \mathrm{LD}} \mathbf{R}_{\mathrm{HO}}(\mathbf{t}) \ldots . .(\mathrm{i}) \\
& \mathbf{R}_{\mathrm{HO}}(\mathbf{t})=V \text { satLsat } \int_{\text {Vsat }(\mathrm{t}-\Omega \mathrm{t})}^{\mathrm{Vsatt}} \text { DlVsattdt....(ii) } \\
& \mathrm{C}_{\text {PatHO-LEO }}(\mathrm{t})=\mathrm{M} \cdot \mathrm{H}_{\mathrm{MN}, \mathrm{LD}}+\mathrm{M} \cdot \mathrm{H}_{\mathrm{AR}, \mathrm{AR}} \mathbf{R}_{\mathrm{HO}}(\mathrm{t}) \boldsymbol{\alpha}+ \\
& \left\{\mathrm{M} \cdot \mathbf{H}_{\mathrm{AR}, \mathrm{AR}}(\mathrm{S}-\mathbf{1})+\mathrm{M} \cdot \mathrm{S}\right\} \times \mathbf{n}(\mathrm{t})(\mathbf{1}-\boldsymbol{\alpha}) \lambda \ldots \text { (iii) }
\end{aligned}
$$

Now here if we can control any one of the parameters of the above equation (i) \& (iii) we can also control the whole cost. In this paper we have proposed an idea to control the number of hops between the MN and the location directory. For that we have generated the idea of implementing a wideband receiver at the receiving end. Using this we can control the number of hops of the network. Here we have made $\mathbf{H}_{\text {MN,LD }}$ $=2$.For that we have used a dual hopping wideband receiver.

\section{Dual-Hopping Wideband Receiver}

An example of wideband receiver front-end architecture is shown in Figure 6 [18]. In the front-end of a receiver we will found the band pass filters (BPF), and the voltage-controlled oscillator (VCO). Wide tuning Range is desirable for these building blocks to be reconfigurable across a broad spectrum. In addition to reconfiguration, high quality factor is desired for low insertion loss and narrow-bandwidth filters. High quality factor also helps to lower the power consumption of the entire front-end.

Figure 7shows the dual-hop architecture. The input spectrum at the antenna ranges from megahertz to gigahertz. A narrow band of this wide input spectrum is filtered by the band-pass filter, removing most distant interferers [19]. This band is amplified through the low-noise amplifier (LNA) before down-conversion through the mixer using the local oscillator signal from a wide-range synthesizer (VCO). The filter and synthesizer are controlled by the same voltage $\mathrm{VC}$ and hop within the input band in unison. The reconfigurable VCO and filter hop covers the input spectrum and selects bands wider than the final, desired signal bandwidth and is therefore termed as the "coarse hop". The VCO hop-step is set by a consideration of both the minimum achievable bandwidth of the filter, and also the minimum VCO hop resolution achievable with low power. Due to the limitation on the minimum achievable hop-step, a second stage hop implemented using a mixer-filter array is required to select the signal band.

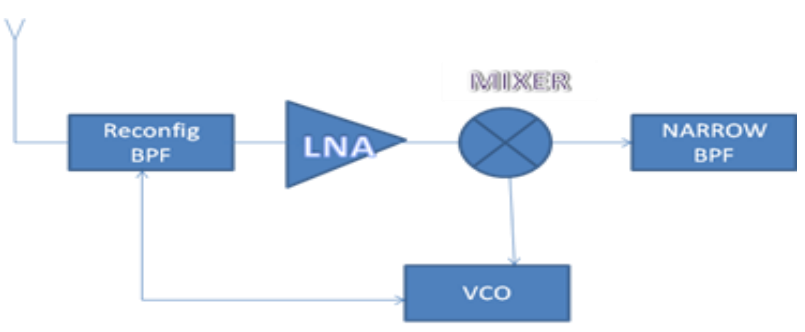

Wideband front-end architecture.

Figure 6: Wideband front-end architecture

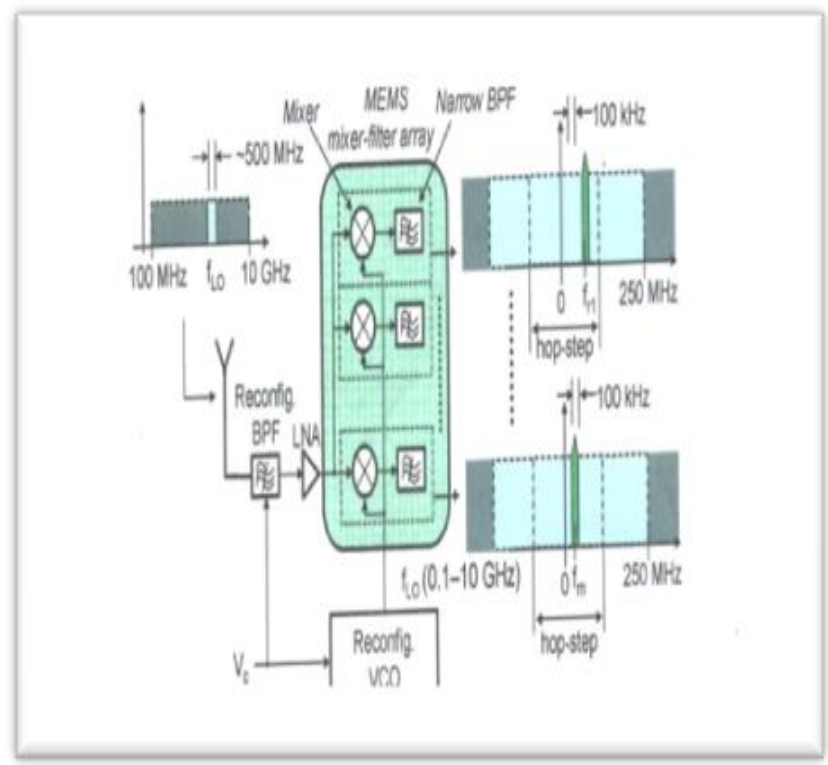

Figure7. Dual Hop architecture

The mixer is implemented as a micromechanical resonator. MEMS resonators can perform both mixing and filtering. In the proposed design, a fine-hopping of about $100 \mathrm{kHz}$ is set by the signal band. The mechanical resonance of the beam resonator performs the filtering operation, and can be designed to extract the $100-\mathrm{kHz}$ signal band from the coarse band.

Since the mechanical mixer is small in area, fine-hopping is performed by designing an array of mixer-filters, each having a different mechanical resonance, to filter different $100-\mathrm{kHz}$ signal bands. Fine hopping is done by selecting between the outputs from the array. A dual-hop architecture is necessary to achieve the desired operation described - while coarsehopping allows for coverage of a wide frequency spectrum, it is relatively slow. The electric switching between mixer 
outputs in fine-hopping is relatively fast (on the order of nanoseconds), and compensates for the relatively slow (on the order of milliseconds) coarse-hopping.

Now, if we design a receiver with different number of hops we will get different cost. Thus we can control the cost by limiting the number of hops.

\section{SIMULATION RESULTS:}

Here we have done our simulation using MATLAB 7. Here we have evaluated the cost of MIP and PatHOLEO based on the following simulation results constraining the number of hops to 2 .

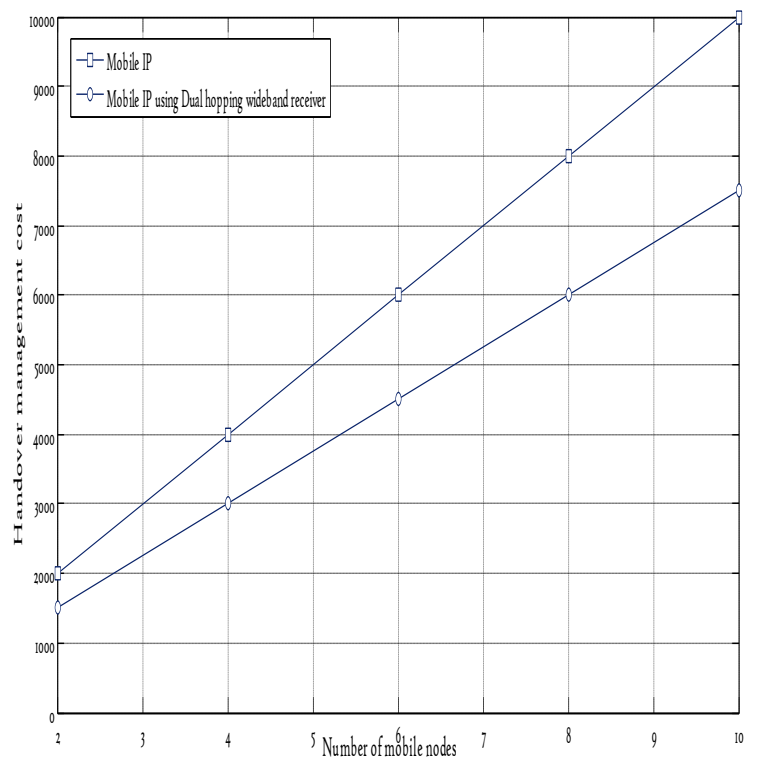

Figure8:Handover management cost of Mobile IP

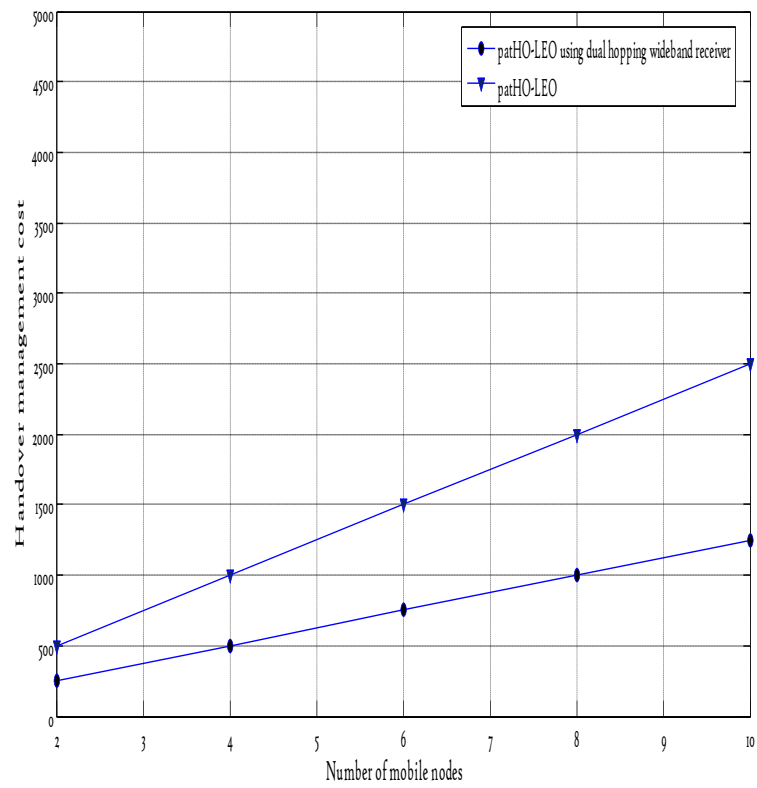

Figure9: Handover management cost of PatHO-LEO
In this paper figure 8 represents the evaluation of handover management cost of Mobile IP. The figure signifies constraining the frequency hops and using a dual hopping wideband receiver mobility cost of MIP gets reduced and it only depends on the message size and $\mathrm{R}_{\mathrm{HO}}$

So, the equation becomes

$\mathrm{C}_{\mathrm{MIP}}(\mathrm{t})=\mathrm{M}$.2. $\mathrm{R}_{\mathrm{HO}}(\mathrm{t}) \ldots(\mathbf{5})$

From equation (5) we can come to an conclusion that handover management cost of Mobile IP depends upon only the message size and rate of handover occurrence $\mathrm{R}_{\mathrm{HO}}(\mathrm{t})$

In accordance with Mobile IP handover management cost PatHO-LEO handover management cost also gets reduced .This has been evaluated in the above figure9.

Here we have evaluated the handover management cost for both MIP and PatHO-LEO on the basis of the number of mobile nodes. From [3] we can find out that the handover management cost of MIP is higher. Now here based on our proposed work, we have evaluated the cost separately for MIP and PatHO-LEO.

As $\mathbf{C}_{\text {PatHO-LEO }}(t)=M .2+M . H_{\mathrm{AR}, \mathrm{AR}} \mathbf{R}_{\mathrm{HO}}(\mathrm{t}) \alpha+$

$\left\{\mathbf{M} \cdot \mathbf{H}_{\mathrm{AR}, \mathrm{AR}}(\mathbf{S}-\mathbf{1})+\mathbf{M} \cdot \mathbf{S}\right\} \times \mathbf{n}(\mathrm{t})(\mathbf{1}-\alpha) \lambda$

We have got the simulation results which shows that the cost is reduced from 10000 to 7500 for $10 \times 10^{6}$ nodes for MIP and for PatHO-LEO for the same number of mobile nodes it has been reduced from 2500 to 1250 .

Hence we can conclude that our proposed work gives a better approach to deal with the handover management cost for both the network.

\section{CONCLUSION}

In this paper we have proposed a new method for the mobility management cost of PatHO-LEO and Mobile IP. Relaying on the simulation results we showed that our proposed mechanism reduced the handover management cost of both the network from the previous results.

\section{FUTURE WORK}

In future we can use frequency hopping filter for varying the hops not only between MN and local directory, but also for the adjacent satellites. We can also focus on the occurrence rate of paging to reduce the cost of PatHO-LEO and also the method of speed control for controlling the cost for both MIP and PatHO-LEO.

\section{REFERENCES}

[1] S. L. Kota, P. A. Leppanen, and K. Pahlavan, Broadband Satellite Communications For Internet Access, Kluwer Academic Publishers, 2004.

[2] A. Jamalipour, "Satellites in IP networks," in Wiley Encyclopaedia of Telecommunications, vol. 4, Wiley, 2002, pp. 2111-2122.

[3] L. Strand, "Linux mobile IPv6 HOWTO," Apr. 2004.

[4] P. Bhagwat, C. Perkins, and S. Tripathi, "Network layer mobility: An architectureand survey," IEEE Pers. Commun., vol. 3, no. 3, pp. 54-64, June1996. 
[5] Endler, M., Nagamuta, V. "General approaches for implementing seamless handover" Proceedings of the second ACM international workshop on Principles of mobile computing", p.17-24, 2002.

[6] E. Cayirci and I. F. Akyildiz, "User mobility pattern scheme for locationupdate and paging in wireless systems," IEEE Trans. Mobile Computing, vol. 1, no. 3, pp. 236-247, 2002.

[7] Debabrata Sarddar, Shubhajeet Chatterjee, Ramesh Jana, Shaik Sahil Babu, Hari Narayan Khan, Utpal Biswas and M.K.Naskar, "Fast Handoff Implementation by using Curve Fitting Equation With Help of GPS", International Journal of Computer Science issues (IJCSI) Vol. 08, Issue 03, pp. 535-542, No. 1, May 2011, ISSN (Online): 1694-0814.

[8] Debabrata Sarddar, Shubhajeet Chatterjee, Ramesh Jana, Hari Narayan Khan, Shaik Sahil Babu, Hari Narayan Khan, Utpal Biswas and M.K.Naskar, "Minimization of Handoff Latency by Vector Analysis Method", International Journal of Computer Science issues (IJCSI) Vol. 08, Issue 03, pp. 570-576, No. 1, May 2011, ISSN (Online): 1694-0814.

[9] Debabrata Sarddar, Shubhajeet Chatterjee, Ramesh Jana, Shaik Sahil Babu, Hari Narayan Khan, Utpal Biswas and M.K.Naskar, " Minimization of Handoff Latency by Distance Measurement Method", International Journal of Computer Science issues (IJCSI) Vol. 08, Issue 02, pp. 283-289, March 2011, ISSN(Online): 1694-0814.

[10] Debabrata Sarddar, Joydeep Banerjee, Tapas Jana,Souvik Kumar Saha, Utpal Biswas and M. K. Naskar, "Minimization of Handoff Latency by Angular Displacement Method Using GPS Based Map", International Journal of Computer Science Issues (IJCSI), Vol. 7, Issue 3, No 7, pp. 29-37, May 2010 ISSN (online): 1694-0784. ISSN (print): 1694-0814.

[11] P. K. Chowdhury, M. Atiquzzaman, W. Ivancic ,Handover Schemes in Satellite Networks: State-of-the-art and future research directions IEEE communications surveys 4TH Quarter 2006, Vol. 8, NO. $4 \mathrm{~A}$

[12] C. Perkins, "IP mobility support," RFC 2002, IEFT, Oct. 1996, available at http://www.ietf.org/rfc/rfc2002.txt.

[13] T"uys"uz and F. Alag"oz, "Satellite mobility pattern based handover management algorithm in LEO satellites," in Proc. IEEE ICC 2006, Istanbul,Turkey, June 2006.

[14] Ays, eg"ul T"uys"uz and Fatih Alag"oz, "Satellite Mobility Pattern Scheme for centrical and Seamless Handover Management in LEO Satellite Networks", JOURNAL OF COMMUNICATIONS AND NETWORKS, VOL. 8, NO. 4, DECEMBER 2006.

[15] M.Atiquzzaman, S. Fu, and W. Ivancic, "TraSH-SN: A transport layer seamless handoff scheme for space networks," in Proc. ESTC 2004, Palo Alto, CA, June 2004.

[16] X. Zhang, J. G. Castellanos, and A. T. Campbell, "Design and performance of mobile IP paging," ACM Mobile Networks and Appl., vol. 7, no. 2, Mar.2002.

[17] A. G. Valko, "Cellular IP-a new approach to Internet host mobility," ACM Computer Commun. Rev., Jan. 1999
[18] Design and Characterization of a RFFrequency-Hopping Filter by Deepa Parvathy Ramachandran

[19] R. Gharpurey, "Design Considerations in Wireless FrontEnds", http://engr.smu.edu/orgs/ssc/slides/20010130.pdf, Texas Instruments, Inc.

[20] H. N. Nguyen, S. Lepaja, J. Schuringa, and H. R. Van As, "Handover management in low earth orbit satellite IP networks," IEEE Global Telecommunications Conference, San Antonio, TX, USA, pp. 2730-2734, 2529 November 2001.

[21] E. Papapetrou and F.-N. Pavlidou, "QoS handover management in LEO/MEO satellite systems," Wireless Personal Communications, vol. 24, no. 2, pp. 189-204, February 2003.

[22] Debabrata Sarddar, Shubhajeet Chatterjee, Ramesh Jana, Shaik Sahil Babu, Hari Narayan Khan, Utpal Biswas and M.K.Naskar, "Fast Handoff Implementation by using Curve Fitting Equation With Help of GPS", International Journal of Computer Science issues (IJCSI) Vol. 08, Issue 03, pp. 535-542, No. 1, May 2011, ISSN (Online): 1694-0814.

[23] Debabrata Sarddar, Shubhajeet Chatterjee, Ramesh Jana, Hari Narayan Khan, Shaik Sahil Babu, Hari Narayan Khan, Utpal Biswas and M.K.Naskar, "Minimization of Handoff Latency by Vector Analysis Method", International Journal of Computer Science issues (IJCSI) Vol. 08, Issue 03, pp. 570-576, No. 1, May 2011, ISSN (Online): 1694-0814.

[24] Debabrata Sarddar, Shovan Maity, Arnab Raha, Ramesh Jana, Utpal Biswas, M.K. Naskar "A RSS Based Adaptive Hand-Off Management Scheme In Heterogeneous Networks Received signal strength", IJCSI International Journal of Computer Science Issues, Vol. 7, Issue 6, November 2010, pp. $232-239$.

[25] Debabrata Sarddar Papri Mani Utpal Biswas M.K.Naskar, "Fast Handoff Mechanism in Wireless Local Area Networks 'WLAN' using Neighbor Graph Algorithm" International Journal of Computer Applications (0975 - 8887) Volume 25-No.9, July 2011

[26] Debabrata Sarddar, Utpal Biswas Mrinal Kanti Naskar Karmajyoti Panigrahi Pulak Mazumder Arnab Raha and Shubhajeet Chatterjee, "Improved Handoff Efficiency with the help of Neighbour Graph using Carrier to Interference Ratio" International Journal of Computer Applications (0975 - 8887) Volume 27- No.1, August 2011

[27] Debabrata Sarddar, Arnab Raha, Tapas Jana, Shovan Maity, Utpal Biswas, and M.K. Naskar, "Reduction of Error in Handoff Initiation Time Calculation for NextGeneration Wireless Systems" (IJCSE) International Journal on Computer Science and Engineering Vol. 02, No. 06, 2010, 2047-2052

\section{AUTHORS PROFILE}

Debabrata Sarddar is currently pursuing his $\mathrm{PhD}$ at Jadavpur University. He completed his M.Tech in Computer Science \& Engineering from DAVV, Indore in 2006, and his B.Tech in Computer Science \& Engineering from Regional Engineering College, Durgapur in 2001. His research interest includes wireless and mobile system. 
Soumya Das, son of Mr. Subrata Das and Mrs. Swapna Das, currently pursuing his B.Tech Degree in Electronics \& communication engg. at Bengal Institute of Technology under West Bengal University of Technology. His research interest includes mobile communication \& satellite communication.

Dipsikha Ganguly currently pursuing B.Tech Degree in Electronics \& communication engineering at B.P.Poddar Institute of Management \& Technology under West Bengal University of Technology. Her research interest includes wireless communication \& satellite communication.

Kalyan Kumar Das completed his BE degree in Computer Science and Engineering from REC (NIT), Durgapur in 2002 and M. Tech. in Computer Technology from Jadavpur University in 2011. His research interest includes wireless sensor networks and wireless communication systems.

Sougata Chakraborty is presently an employee of IBM (Pune). He has completed his B.Tech in IT from Murshidabad College of Engineering and Technology and M Tech degree on computer science \& engineering from Jadavpur University on 2011. His research interest includes wireless sensor networks and wireless communication systems.

Kunal Hui is presently an employee of L\&T (Mumbai). He has completed his B.Tech in Computer Science \& Engineering from Murshidabad College of Engineering and
Technology(2004 -2008) and M.Tech in Computer Science \& Engineering from Haldia Institute of Technology(2009-2011). His research interest includes wireless sensor networks and wireless communication systems.

Mrinal Kanti Naskar received his B.Tech. (Hons) and M.Tech degrees from E\&ECE Department, IIT Kharagpur, India in 1987 and 1989 respectively and Ph.D. from Jadavpur University, India in 2006.. He served as a faculty member in NIT, Jamshedpur and NIT, Durgapur during 1991-1996 and 1996-1999 respectively. Currently, he is a professor in the Department of Electronics and Tele-Communication Engineering, Jadavpur University, Kolkata, India where he is in charge of the Advanced Digital and Embedded Systems Lab. His research interests include ad-hoc networks, optical networks, wireless sensor networks, wireless and mobile networks and embedded systems. He is an author/co-author of the several published/accepted articles in WDM optical networking field that include "Adaptive Dynamic Wavelength Routing for WDM Optical Networks" [WOCN,2006], "A Heuristic Solution to SADM minimization for Static Traffic Grooming in WDM uni-directional Ring Networks" [Photonic Network Communication, 2006], "Genetic Evolutionary Approach for Static Traffic Grooming to SONET over WDM Optical Networks" [Computer Communication, Elsevier, 2007], and "Genetic Evolutionary Algorithm for Optimal Allocation of Wavelength Converters in WDM Optical Networks" [Photonic Network Communications,2008]. 